\title{
A new experimental ground vehicle with hybrid control and hybrid vision sensor
}

\author{
J. M. Rendón-Mancha*¹, G. Sanahuja ${ }^{2}$, P. Castillo ${ }^{2}$, R. Lozano ${ }^{2}$ \\ ${ }^{1}$ Facultad de Ciencias, UAEM,Cuernavaca, México \\ *rendon@uaem.mx \\ 2 Heudiasyc Laboratory, UMR CNRS 6599, \\ Université de Technologie de Compiègne, Compiègne, France
}

\begin{abstract}
This paper presents a new hybrid control algorithm based on saturation functions and its real-time application to a ground vehicle. The hybrid control is developed from a nonlinear continuous control law and the objective is to obtain the optimal sampling period $T$ to apply the controller in real experiences. The stability analysis was made in discrete time. The experimental platform is composed of a remote control toy car and a vision system. The vision system is built using a simple webcam and a diode laser. This system is fast, accurate, inexpensive and easy to implement. Simulations and experiments show the stability and robustness of the closed-loop system. The proposed control law performance is compared with a linear control algorithm.
\end{abstract}

Keywords: Nonlinear control, saturation functions, computer vision, experimental platform, laser.

\section{RESUMEN}

Este artículo presenta un nuevo algoritmo de control híbrido basado en funciones de saturación y su implementación en tiempo real en un vehículo terrestre. El control híbrido fue desarrollado a partir de una ley de control continua no lineal y el objetivo es obtener el período de muestreo óptimo $T$ para aplicar el controlador en experimentos. La plataforma experimental se compone de un carro de juguete a control remoto y de un sistema de visión. El sistema de visión se construye usando una webcam y un diodo láser. El sistema es rápido, preciso, barato y fácil de implementar. Las simulaciones y los experimentos muestran la estabilidad y la robustez del sistema de lazo cerrado. Se realiza una comparación del desempeño de la ley de control propuesta con la de un algoritmo de control lineal.

\section{Introduction}

The problems arising in the implementation of continuous time control laws have attracted a growing interest among the research community. The fact that, in practice, every control input of a system is always limited motivates the study of the stabilization of linear control systems with bounded inputs. For exponentially unstable systems, i.e., systems with poles in the open right half-plane, only locally stabilizing controllers can be obtained; therefore, globally stable control systems can only be obtained for integrators in cascade or in general for null controllable systems [1].

The stabilization of linear systems with bounded controls has been studied since the seventies. Indeed, Fuller [2] showed that it is not possible to globally stabilize three or more integrators connected in cascade by simply saturating a stabilizing state feedback control law. Later, Sussmann et al. [1] showed this constraint can be extended to systems having three or more eigenvalues on the $j w$-axis. Teel [3] proposed a control algorithm that achieves global asymptotic stabilization of a chain of $n$ integrators. The control strategy is based on a series of nested saturation functions.

Sussmann et al. [1] generalized Teel's idea in 1994 to the case of continuous-time null controllable systems. Then, Johnson and Kannan [4] provided an approach that allows the designer to pick transformations that facilitate the placement of the closed-loop poles. Yang et al. [5] proposed the discrete-time counterpart of Sussmann's extension. This approach is iterative and constructive and requires several matrix transformations that have to be computed. Note that Marchand and Hably [6] improved the performance of the nonlinear control law proposed in [1] for a continuous-time chain of integrators by introducing variable saturation levels. They also 
provided numerical comparisons between several techniques based on bounded inputs in order to measure the convergence speed of the algorithms.

Lin and Saberi [7] proposed a linear controller that achieves semi-global stabilization for nullcontrollable linear systems using state feedback. In 1995, the same authors [8] proposed a semi-global stabilization scheme for the discrete-time case by using a linear controller that achieves pole placement. In [9], an alternative way to semiglobally stabilize a continuous-time linear system having poles in the closed LHP with amplitude constrained inputs is proposed. The discrete-time counterpart is presented in [10].

In addition, only few control results have been tested in real experiences, for example Castillo et al. [11] showed experimentally, on a quad-rotor mini-aircraft platform, that the optimal control is less efficient than a bounded controller based on Teel's nested saturations.

A common situation in real-time experiences is to apply the control law in a PC or micro controller, this is, the control algorithm is constructed in a way that it holds the analog signal constant until a new conversion is commanded. It is then natural to choose the sampling instants $t_{k}$ as the times when the control changes; then, to apply this control strategy in a real-time experience, we need to obtain the discrete-time model of the system, but we need also to prove that the closed-loop system remains stable.

In this paper, we address the problem of obtaining the optimal sampling period $T$ to assure that the closed loop system remains stable when implementing non-linear control algorithms in real experiences. On the other hand, we present also a new low-cost camera-laser vision sensor to measure the distance using a simple webcamera and a diode laser. The new sensor is designed to be used in the real experiences to provide the distance feedback to our control.

Range sensors based on lasers have been developed since the seventies, a review can be found in [12]. Many companies offer industrial 3D scanners, see [13], most of them use laser-based techniques. Even though these scanners are widely used, research on range sensors using structured light remains very active [14]. Industrial scanners are normally expensive. A low-cost technique uses one or more laser sources and a camera to triangulate laser projections in order to estimate the three-dimensional structure of objects [15].

Recently, control systems rely increasingly on vision systems as input. This is mainly due to the fact that vision systems have become smaller, faster, reliable and inexpensive. Some control strategies use fixed cameras, which are external to the plant, to implement different tasks, it is the case of manipulator arms [16]. Mobile robots can also be controlled using externally fixed cameras where the control is constrained to the field of vision of the cameras. In order to provide autonomy to the robots, all sensors must be embedded in the robot.

A vision module must provide the control system with useful information to decide how to move. This information can be classified in threedimensional data, image measurements in $2 \mathrm{D}$ or a combination of them [17] [18]. Three-dimensional information, such as points, vectors and rotation matrices, is used to situate the robot in a $3 \mathrm{D}$ world. On the other hand, using image measurements means that the vision system delivers data computed from the image which is sufficient to control the robot but that is not 3D information. Control strategies using the first or the second type of information have been traditionally treated with names such as "look and move" vs. visual servoing [19], [20], camera space manipulation [16] and others. Control strategies using image measurements can sometimes improve accuracy avoiding redundant computations and also resulting in faster algorithms.

In this work, three-dimensional information is used to control an autonomous robot. A distance defined into a three-dimensional world is estimated using a vision sensor. The distance of the vehicle to an object in front of it is computed using a camera/laser simplified range sensor. This distance is used in a closed-loop system to keep the vehicle at a specified distance from the object. The experimental platform used is composed of a toy car equipped with a webcam and a diode laser. The vision sensor must have a fast response in order to work in the closed-loop system. 


\section{A new experimental ground vehicle with hybrid control and hybrid vision sensor, J. M. Rendón-Mancha et al., 310-322}

Therefore, the goals of this paper are

- To present a new idea to obtain the optimal sampling period $T$ to assure that the closed-loop system to implement nonlinear controllers in real experiences remains stable.

- To present a low-cost camera-laser vision sensor to measure the distance using a simple webcam and a diode laser.

This paper is organized as follows: Section 2 explains the dynamic model of the vehicle. The hybrid control is developed in Section 3, stability is proved in discrete time and some simulations are shown. The camera-laser sensor is described in Section 4. Experimental results appear in Section 5. Finally, Section 6 is reserved to conclusions.

\section{Dynamic model of the vehicle}

The dynamical equation of the cart in the $x$-axis may be obtained by setting it out in 2D, with two identical wheels and a chassis, see Figure 1. We will denote $m$ the mass of each wheel, $C_{i}$, its center (which is assumed to be also its center of gravity), $r$ its radius and $J$ its moment of inertia. For the chassis we will denote $M$ its mass and $L$ the distance between wheels. The center of gravity of the chassis is assumed to be on its middle. The contact between ground and wheels is assumed to be a point, and the study will be done in the case of limiting friction for each wheel.
Each wheel $i, \forall_{i}=1,2$, is subjected to the following forces:

1. Its weight $P_{i}=m g$

2. The reaction force, $R_{i}$

3. The force, $F_{c h / i}$, from the chassis applied to the axis of the wheel.

Under the hypothesis of limiting friction and from Figure 1 , we have that, $\vec{R}_{i}=F_{f, i} \cdot \vec{x}+N_{i} \cdot \vec{z}$, where $F_{f, i}$ is the tangential force and $N_{i}$ is the normal force. Moreover, $F_{f, i} \approx \mu N_{i}$, with $\mu$ the coefficient of friction between wheel and ground. The torque applied to the axis of the driving wheel is denoted by $\tau$. In addition, the chassis is subjected to its weight $P=M g$, applied in its center. Thus, from Figure 1 and employing the Newton approach, it yields for the driving wheel:

$$
\begin{aligned}
& \mu N_{1}-\overrightarrow{F_{c h / 1}} \cdot \vec{x}=m \ddot{x} \\
& -m g-\overrightarrow{F_{c h / 1}} \cdot \vec{z}+N_{1}=0 \\
& \tau-r \mu N_{1}=\frac{J}{r} \ddot{x}
\end{aligned}
$$

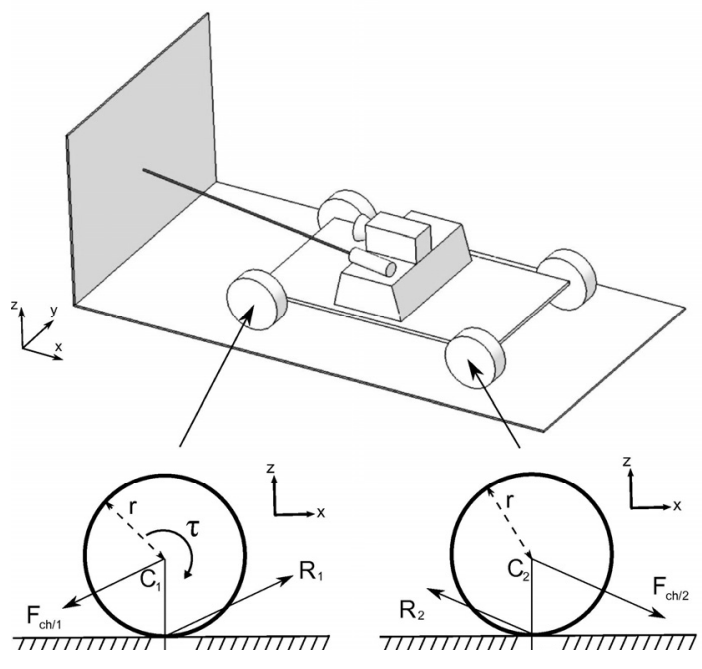

Figure 1. Scheme of the cart. 


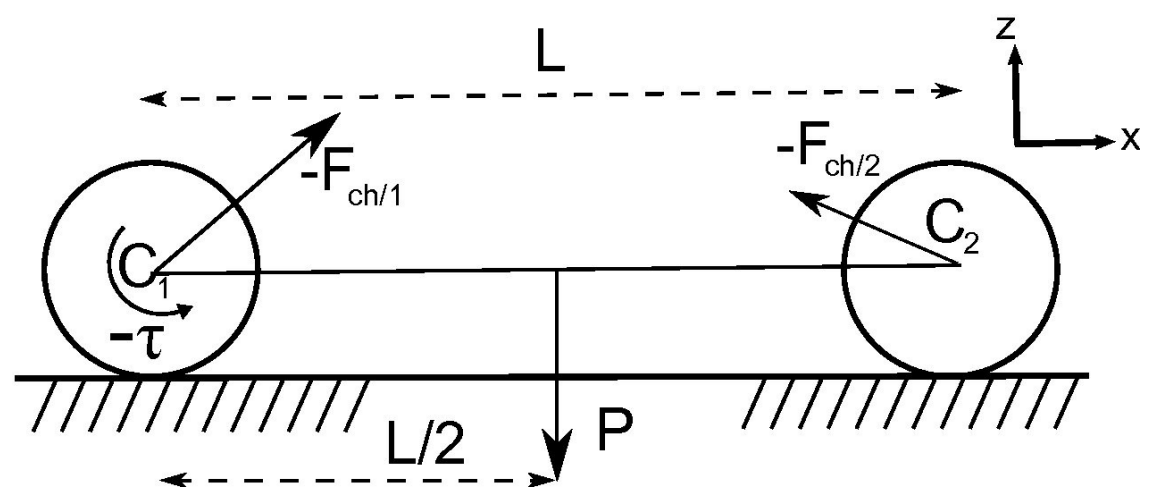

Figure 2. Chassis of the cart.

The dynamic for the free wheel is described by,

$$
\begin{aligned}
& -\mu N_{2}+\overrightarrow{F_{c h / 2}} \cdot \vec{x}=m \ddot{x} \\
& -m g-\overrightarrow{F_{c h / 2}} \cdot \vec{z}+N_{2}=0 \\
& r \mu N_{2}=\frac{J}{r} \ddot{x}
\end{aligned}
$$

From Figure 2, the chassis equations are

$$
\begin{aligned}
& \overrightarrow{F_{c h / 1}} \cdot \vec{x}-\overrightarrow{F_{c h / 2}} \cdot \vec{x}=M \ddot{x} \\
& -M g+\overrightarrow{F_{c h / 1}} \cdot \vec{z}+\overrightarrow{F_{c h / 2}} \cdot \vec{z}=0 \\
& -\tau-L \overrightarrow{F_{c h / 2}} \cdot \vec{z}+M g \frac{L}{2}=0
\end{aligned}
$$

After some algebraic manipulations using (1)-(9), we obtain,

$$
\ddot{x}=k_{\tau} \tau
$$

with

$$
k_{\tau}=\frac{1}{2 m r+M r+2 \frac{J}{r}}
$$

Note that, (10) is the classical mathematical equation of a cart moving along the $x$-axis, the friction terms being neglected. Furthermore, the robustness of the control strategy will be proved in real time experiences where the friction is present.

The control law applied to the cart is based on saturation functions; stability of the closed-loop system for the continuous time was proved in [21]; however, the control strategy will be computed in discrete time and its stability in this case is not proved yet, thus, the proposed control law has the form:

$$
k_{\tau} \tau=-\sigma_{a}\left(\dot{x}+\sigma_{b}\left(\dot{x}+\left(x-x_{d}\right)\right)\right)
$$

Where $x_{d}$ is the desired value of the position, $a, b>0$ are constant and $\sigma_{j}\left(f_{x, x}\right)$, with $j=a, b$, is a saturation function such that

$$
\sigma_{j}\left(f_{\dot{x}, x}\right)=\left\{\begin{array}{cc}
j, & f_{\dot{x}, x}>j ; \\
f_{\dot{x}, x}, & -j \leq f_{\dot{x}, x} \leq j ; \\
j, & f_{\dot{x}, x}<-j .
\end{array}\right.
$$

And $f_{x, x}$ is a function of $\dot{x}$ and $x$. For further analysis, we will use $\bar{\tau}=k_{\tau} \tau$ as the control input.

\section{Hybrid control}

As mentioned before, in real-time experiences the control law is applied in a PC or micro controller and it is natural to choose sampling instants, $t_{k}$, as the times when the control changes. In this section, the optimal sampling period $T=t_{k}-t_{k-1}$ is 


\section{A new experimental ground vehicle with hybrid control and hybrid vision sensor, J. M. Rendón-Mancha et al., 310-322}

obtained to assure that the closed-loop system remains stable when implementing the control algorithm in real-time experiments. For further analysis, we will make $x_{d}=0$.

Let us define the following functions:

$$
\begin{aligned}
& z_{1}=\dot{x}+x \\
& z_{2}=\dot{x}
\end{aligned}
$$

then,

$$
\dot{z}_{1}=\bar{\tau}+z_{2} .
$$

Making a new system given by (14) and (10) and rewriting them in terms of $z_{i}$, we get

$$
\begin{aligned}
& \dot{z}_{1}=z_{2}+\bar{\tau}, \\
& \dot{z}_{2}=\bar{\tau}
\end{aligned}
$$

or in the classical form:

$$
\dot{\bar{z}}=A \bar{z}+B \bar{\tau}
$$

With $\bar{z}=\left[\begin{array}{l}Z_{1} \\ z_{2}\end{array}\right] A=\left[\begin{array}{ll}0 & 1 \\ 0 & 0\end{array}\right]$ and $B=\left[\begin{array}{l}1 \\ 1\end{array}\right]$

The discrete-time model of (16) is

$$
\bar{z}_{k+1}=A_{d} \bar{z}_{k}+B_{d} \bar{\tau}_{k}
$$

where

$$
B_{d}=\int_{0}^{T} e^{A s} B d s
$$

Exact discretization may sometimes be intractable due to the heavy matrix exponential and integral operations involved. It is much easier to calculate an approximate discrete model, based on that for small time-steps. The approximate solution then becomes

$$
\begin{gathered}
A_{d}=e^{A T} \approx I+A T \\
\int_{0}^{T} e^{A s} B d s \approx\left(I T+\frac{1}{2} A T^{2}\right) B
\end{gathered}
$$

with I being the identity matrix. Consequently,

$$
A_{d}=\left(\begin{array}{ll}
1 & T \\
0 & 1
\end{array}\right)
$$

and

$$
B_{d}=\left(\begin{array}{c}
T+\frac{T^{2}}{2} \\
T
\end{array}\right)
$$

Rewriting (17),

$$
\begin{aligned}
& z_{1_{k+1}}=z_{1_{k}}+T z_{2_{k}}+T\left(1+\frac{T}{2}\right) \bar{\tau}_{k} \\
& z_{2_{k+1}}=z_{2_{k}}+T \bar{\tau}_{k}
\end{aligned}
$$

the discrete time form of the control input (11) is

$$
\bar{\tau}_{k}=-\sigma_{a}\left(z_{2_{k}}+\sigma_{b}\left(z_{1_{k}}\right)\right),
$$

using the above into (20), it yields

$$
z_{2_{k+1}}=z_{2_{k}}-T \sigma_{a}\left(z_{2_{k}}+\sigma_{b}\left(z_{1_{k}}\right)\right) .
$$

Note from the above that, if $\left|z_{2_{k}}\right|>b$ then

$$
z_{2 k+1}=z_{2 k}(1-T)
$$

this implies that, for all $T<2$, all poles lie inside the unit circle and consequently $z_{2_{k}}$ will decrease. This follows that there exists a $t_{k_{1}}$ such that for all $t_{k}>t_{k_{1}},\left|z_{2_{k}}\right|<b$. Choosing $a=2 b$, we obtain

$$
\begin{aligned}
& \forall t_{k}>t_{k_{1}} \\
& \quad \bar{\tau}_{k}=-z_{2_{k}}-\sigma_{b}\left(z_{1_{k}}\right),
\end{aligned}
$$

From (19) and the above, we obtain

$$
\begin{aligned}
& z_{1_{k+1}}=\quad z_{1_{k}}+T z_{2_{k}}+T\left(1+\frac{T}{2}\right) \bar{\tau}_{k} \\
& =z_{1_{k}}+T z_{2_{k}}-T\left(1+\frac{T}{2}\right)\left[z_{2_{k}}+\sigma_{b}\left(z_{1_{k}}\right)\right] \\
& =\quad z_{1_{k}}-T\left(1+\frac{T}{2}\right) \sigma_{b}\left(z_{1_{k}}\right)-z_{2_{k}}\left(\frac{T^{2}}{2}\right) \\
& =\quad z_{1_{k}}-\left(T+\frac{T^{2}}{2}\right) \sigma_{b}\left(z_{1_{k}}\right)+w_{k}
\end{aligned}
$$


Where $w_{k}=-z_{2_{k}}\left(\frac{T^{2}}{2}\right)$. Notice that, $z_{2_{k}}$ decreases and is bounded, this will imply that, $w_{k}$ will be also quite small; thus, Equation (23) can be rewritten as follows:

$$
\begin{aligned}
z_{1_{k+1}} & =z_{1_{k}}-\left(T+\frac{T^{2}}{2}\right) \sigma_{b}\left(z_{1_{k}}\right) \\
& =z_{1_{k}}\left(1-T+\frac{T^{2}}{2}\right)
\end{aligned}
$$

The above system will be asymptotically stable if all poles lie inside (none is on) the unit circle, or what is the same, all poles have magnitude less than 1 ; therefore, it is necessary that $T+\frac{T^{2}}{2}<2$. To know the values of $T$ to remain the system stable, the previous inequality needs to be solved, thus,

$$
T^{2}+2 T-4<0
$$

solving the above equation, the possible values to make the system stable are for all $T \in] 0,1.2361[$. With the above, it follows that $z_{1_{k}}$ and $z_{2_{k}}$ goes to zero. Consequently, from (13) and (12), $\dot{x}$ and $x$ goes also to zero.

\section{Simulations}

In this section we present the performance in simulation when applying the control strategy (21) into the system (17). We used a pulse of amplitude $15 \mathrm{~cm}$ every 50 sampling instants as the desired position. Figure 3 shows the system response when applying the proposed control algorithm with a sampling period of $T=1$. Note from this figure that the system in this case remains stable. Incrementing the sampling period to $T=2$, the closed-loop system becomes unstable, see Figure 4. This fact is normal because for this case $T$ is outside the range of possible values.

\section{Camera-Laser sensor platform}

The experimental platform (see Figure 5) is composed of an electrical cart moving only along the $x$ axis, a vision-laser sensor and the Matlab XPC target system.

The distance $d$ between the cart and the wall is measured using a low-cost sensor composed of a camera and a laser. The camera is "looking" at a spot projected on the wall by a laser pointer, then

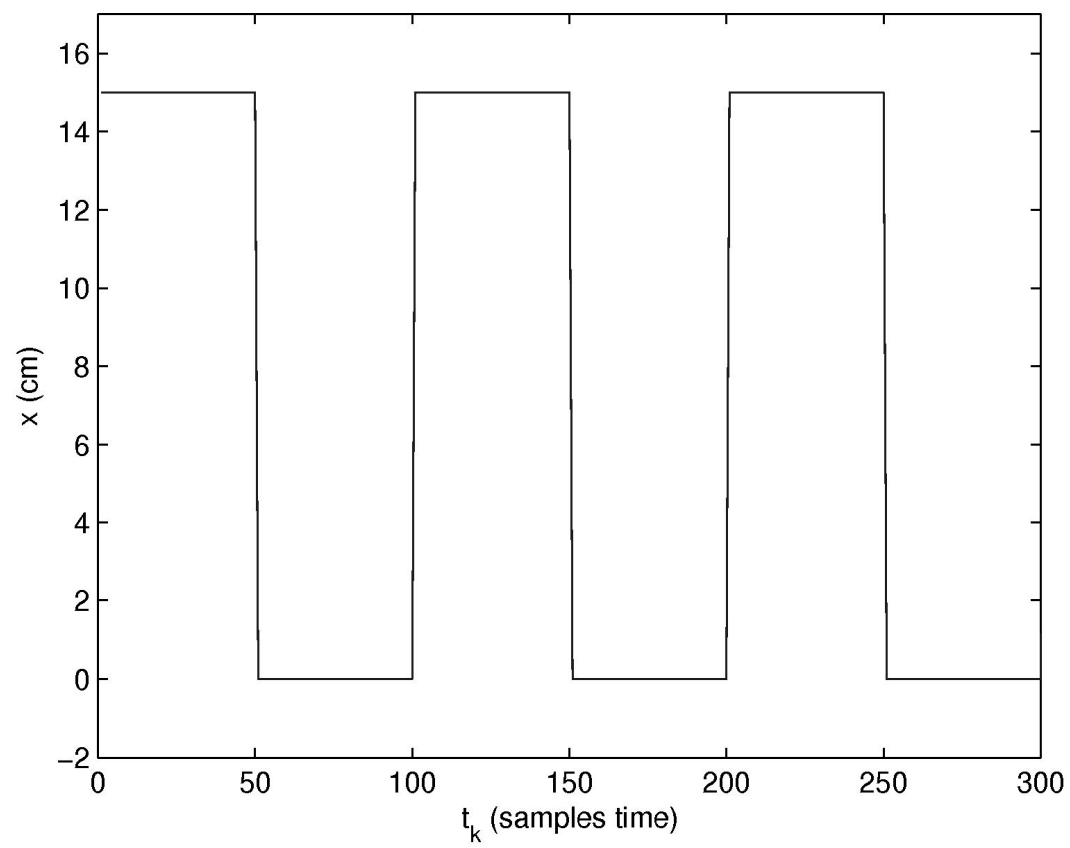

Figure 3. Response of the closed-loop system when applying (21) into (17). The sampling period for this case is $T=1$. 


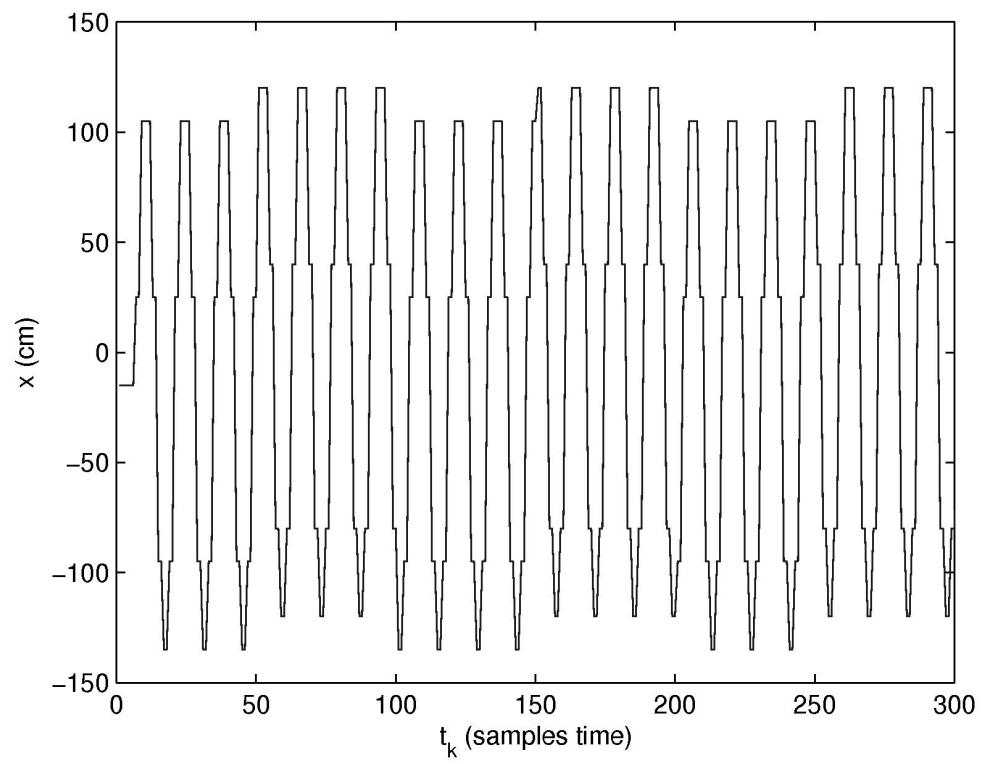

Figure 4. Response of the closed-loop system when applying (21) into (17).

The sampling period for this case is $T=2$.

the image is sent to a computer station (PC vision) to estimate the distance with respect to the camera coordinate system.

The resulting $x$-position is sent to the ground station computer to compute the control inputs using a XPC Target and the RS232 serial communication. The control input is then sent to the motors of the car through an Advantech PCL726 output card. Due to physical constraints in the motors of the cart, the control input signal must satisfy the following inequality:

$O V<\bar{\tau}<5 \mathrm{~V}$

\section{Camera calibration}

We used a calibration technique proposed by Zhang [22] to calibrate the camera. It uses a planar pattern to compute both intrinsic and extrinsic parameters; with this method, we do not need to know the orientations of the pattern, we just need to know its dimensions. First, the intrinsic parameters of the camera are computed and then the extrinsic parameters can be computed for each pattern position. In this work, after calibration of the camera and the laser, all points and vectors are referred to the three-dimensional camera coordinate system.

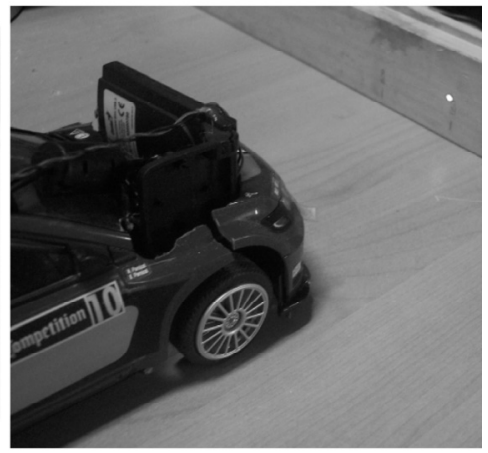

Figure 5. Experimental cart. A webcam and a diode laser pointer were fixed in a remote control toy car. 
The camera intrinsic parameters are represented by the calibration matrix [23]:

$$
K=\left[\begin{array}{lll}
\alpha_{x} & & c_{x} \\
& \alpha_{y} & c_{y} \\
& & 1
\end{array}\right]
$$

and the extrinsic parameters are composed of a rotation matrix and a translation vector, both of them are three-dimensional.

\section{Calibration of the laser}

The aim of this calibration is to find a point $P_{l}$ and a vector $V_{l}$ which define the origin and the direction of the laser in the camera coordinate system. We used a similar technique as for the camera calibration. Once the laser was fixed relatively to the camera, some images of the pattern are taken. In each image, we can easily locate the laser spot position in the pattern $P_{l i}=\left[X_{l i}, Y_{l i}, 0\right]^{T}$ which corresponds to its coordinates in the pattern coordinate system, these points are measured manually. Having the intrinsic parameters of the camera, the extrinsic parameters can be computed for each pattern position (see [22]), these parameters correspond to matrix $R$ and vector $t$. In this way, points can be mapped from the pattern coordinate frame to the camera frame using:

$$
P_{c}=R P_{p}+t
$$

where $P_{p}$ denotes the coordinates of a point in the pattern coordinate system and $P_{c}$ its coordinates in the camera system, thus, the coordinates $p_{l i}=$ $\left[x_{l i}, y_{l i}, z_{l i}\right]^{T}$ of the laser spot in the three-dimensional camera coordinate system are given for each frame by

$$
p_{l i}=R P_{l i}+t
$$

With one pattern image, we can find a possible origin of laser $P_{l}$, and with a minimum of two images, we can define the laser direction $V_{l}$. Using $n$ images, we get $n$ points, a linear regression in 3D-space; the Principal Components Analysis is performed to find the closest line to all of the points. This is done in order to improve precision in the presence of noise (normally imprecisions measuring $P_{l i}$ ).

\section{Computing the distance}

Here, all points and vectors are referred to the three-dimensional camera coordinate system. Once both camera and laser are calibrated, computing the distance between the camera center and the laser spot entails a triangulation problem (same as using stereovision [23]). The goal is to find the intersection between two rays: the one from the laser $\left(P_{l}, V_{l}\right)$ and the one coming from the optical center of the camera passing through the laser spot seen in the image (see Figure 6). The center of the camera is $C=[0,0,0]^{T}$ and the laser projection spot in the image referred to the three-dimensional camera coordinate system is $P_{i}=\left[\left(l_{x}-c_{x}\right) / \alpha_{x},\left(l_{y}-c_{y}\right) / \alpha_{y}, 1\right]^{T}$. Where $\left[l_{x}, l_{y}\right]^{T}$ is the laser projection spot in the image (in pixels) and $\alpha_{x}, \alpha_{y}, c_{x}$ and $c_{y}$ come from the calibration matrix (25).

Usually, due to noise, the two rays do not cross; thus, a simple solution is to choose the midpoint of the common perpendicular to the two rays. This is called the midpoint method. For a comparison of different triangulation methods see [24].

The midpoint of the two rays is the point that minimizes the sum of the distance from the point to each ray. In this way, if each ray is characterized by a normalized vector $\left[a_{i}, b_{i}, c_{i}\right]^{T}$ and a point $\left[x_{i}, y_{i}, z_{i}\right]^{T} i=1,2$, the square distance $d_{i}^{2}$ between a point $\left[x_{0}, y_{0}, z_{0}\right]^{T}$ and the ray $i$ is given by

$$
\begin{gathered}
d_{i}^{2}=x_{0}^{2}\left(b_{i}^{2}+c_{i}^{2}\right)+y_{0}^{2}\left(a_{i}^{2}+c_{i}^{2}\right)+z_{0}^{2}\left(a_{i}^{2}+b_{i}^{2}\right) \\
-2 x_{0} y_{0} a_{i} b_{i}-2 x_{0} z_{0} a_{i} c_{i}-2 y_{0} z_{0} b_{i} c_{i} \\
+2 x_{0}\left(b_{i} k_{i 3}-c_{i} k_{i 2}\right)+2 y_{0}\left(c_{i} k_{i 1}-a_{i} k_{i 3}\right) \\
+2 z_{0}\left(a_{i} k_{i 2}-b_{i} k_{i 1}\right) \\
+k_{i 1}^{2}+k_{i 2}^{2}+k_{i 3}^{2}
\end{gathered}
$$

with

$$
\begin{aligned}
k_{i 1} & =z_{i} b_{i}-y_{i} c_{i} \\
k_{i 2} & =x_{i} c_{i}-z_{i} a_{i} \\
k_{i 3} & =y_{i} a_{i}-x_{i} b_{i}
\end{aligned}
$$

To find the midpoint, we have to minimize the sum $D=d_{1}^{2}+d_{2}^{2}$; thus, differentiating $D$ with respect to $x_{0}, y_{0}$ and $z_{0}$ gives:

$$
\begin{aligned}
\partial D / \partial x_{0}= & \sum_{i=1}^{2} 2 x_{0}\left(b_{i}^{2}+c_{i}^{2}\right)-\sum_{i=1}^{2} 2 y_{0} a_{i} b_{i} \\
& -\sum_{i=1}^{2} 2 z_{0} a_{i} c_{i}+\sum_{i=1}^{2} 2\left(b_{i} k_{i 3}-c_{i} k_{i 2}\right)
\end{aligned}
$$




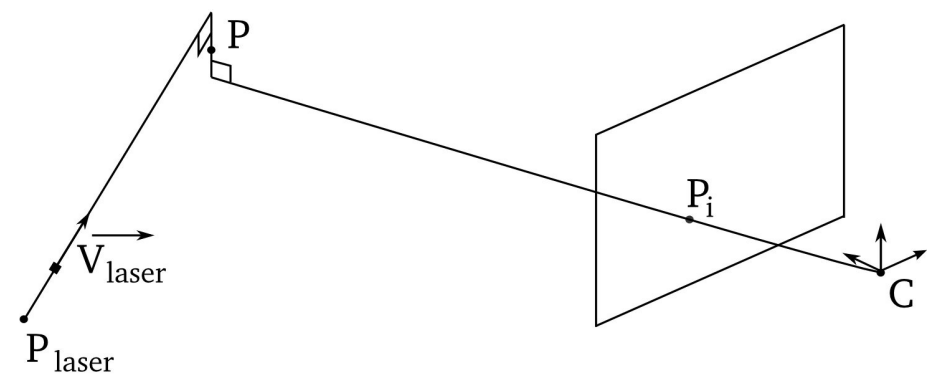

Figure 6. Laser and camera. The midpoint algorithm computes the point between the perpendicular of two rays which is equidistant to the two rays.

$$
\begin{aligned}
\partial D / \partial y_{0}= & \sum_{i=1}^{2} 2 y_{0}\left(a_{i}^{2}+c_{i}^{2}\right)-\sum_{i=1}^{2} 2 x_{0} a_{i} b_{i} \\
& -\sum_{i=1}^{2} 2 z_{0} b_{i} c_{i}+\sum_{i=1}^{2} 2\left(c_{i} k_{i 1}-a k_{i 3}\right)
\end{aligned}
$$

$$
\begin{aligned}
\partial D / \partial z_{0}= & \sum_{i=1}^{2} 2 z_{0}\left(a_{i}^{2}+b_{i}^{2}\right)-\sum_{i=1}^{2} 2 x_{0} a_{i} c_{i} \\
& -\sum_{i=1}^{2} 2 y_{0} b_{i} c_{i}+\sum_{i=1}^{2} 2\left(a_{i} k_{i 2}-b_{i} k_{i 1}\right)
\end{aligned}
$$

To find the minima, we equalize each derivate with zero; then, rewriting (28) to (30) in matrix form gives:

$$
v=B P_{0}
$$

with:

$$
\begin{gathered}
v=\left[\begin{array}{c}
\sum_{i=1}^{2}\left(c_{i} k_{i 2}-b_{i} k_{i 3}\right) \\
\sum_{i=1}^{2}\left(a_{i} k_{i 3}-c_{i} k_{i 1}\right) \\
\sum_{i=1}^{2}\left(b_{i} k_{i 1}-a_{i} k_{i 2}\right)
\end{array}\right] \\
B=\left[\begin{array}{ccc}
\sum_{i=1}^{2}\left(b_{i}^{2}+c_{i}^{2}\right) & -\sum_{i=1}^{2} a_{i} b_{i} & -\sum_{i=1}^{2} a_{i} c_{i} \\
-\sum_{i=1}^{2} a_{i} b_{i} & \sum_{i=1}^{2}\left(a_{i}^{2}+c_{i}^{2}\right) & -\sum_{i=1}^{2} b_{i} c_{i} \\
-\sum_{i=1}^{2} a_{i} c_{i} & -\sum_{i=1}^{2} b_{i} c_{i} & \sum_{i=1}^{2}\left(a_{i}^{2}+b_{i}^{2}\right)
\end{array}\right]
\end{gathered}
$$

Then the midpoint $P_{0}$ is given by

$$
P_{0}=B^{-1} v
$$

In practice, a Gaussian elimination is used to solve (32). To validate the previous algorithms, some manual tests were performed. The vision system was moved along a line and the max error obtained during the test was of two pixels. In addition, the error in the distance measurement depends directly on the distance between the camera and the laser spot.

\section{Experimental results}

In this section, we present the experimental results obtained applying the control strategy (21) and a linear control strategy (PD) to stabilize the cart in a desired position $\left(x_{d}=450 \mathrm{~mm}\right)$. The experiment was performed as follows: the car is located initially at a random position and a fixed desired position, $x_{d}$, is defined. When the car is stabilized in $x_{d}$, we apply several manual perturbations, i.e., when the car reaches the desired value, we move it with the hand, changing its position. This fact leads the vision system to compute the new distance and this information is thus sent to the control algorithm. A new error is then estimated and the control law is recalculated to stabilize the system, see Figures 7-8.

Note from Figure 7 that the linear control law (PD) stabilizes the car in the desired position, but the performance is reduced when applying manual perturbations; it can be seen from the magnitude of the over jumps before the system stabilizes again. Notice also that the control input $\tau_{k}$ has high values. 

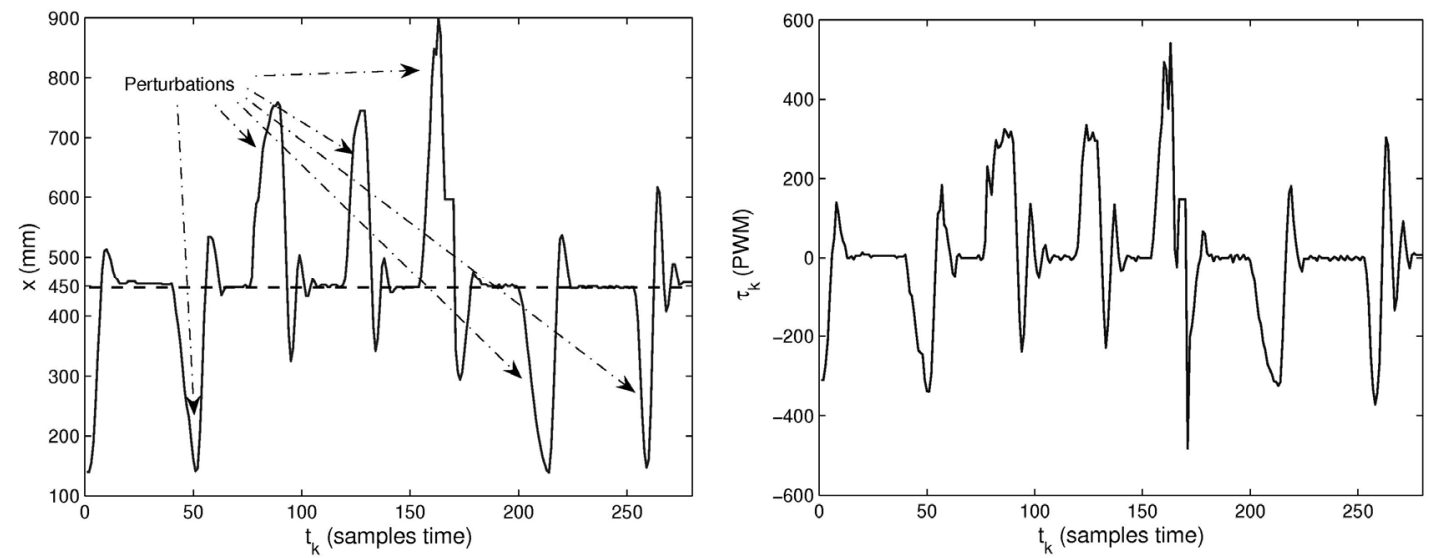

Figure 7. System response of the closed-loop system using a PD control strategy.

From Figure 8, note that when applying the proposed controller, the performance of the system is better. Even in presence of manual perturbations, the performance of the closed-loop system is better than when using a linear controller. In addition, the control input $\tau_{k}$ is smaller and bounded.

\section{Conclusions}

This paper presented a new hybrid nonlinear control based on saturation functions. The system includes a vision sensor composed of a camera and a laser. The model studied is a vehicle moving along one axe only. A discrete-time model has been obtained from the continuous dynamic model and the stability of the closed-loop system was proved. Simulations in discrete-time were performed. The experimental platform developed was composed of an electrical remote control toy car equipped with a low-cost vision sensor. This sensor was developed using a web camera and a diode laser. The vision sensor has a good trade-off between computational cost and precision, being quite fast and accurate to our control purposes. Experimental results in real-time showed that the proposed controller performs better than a classical linear control law.
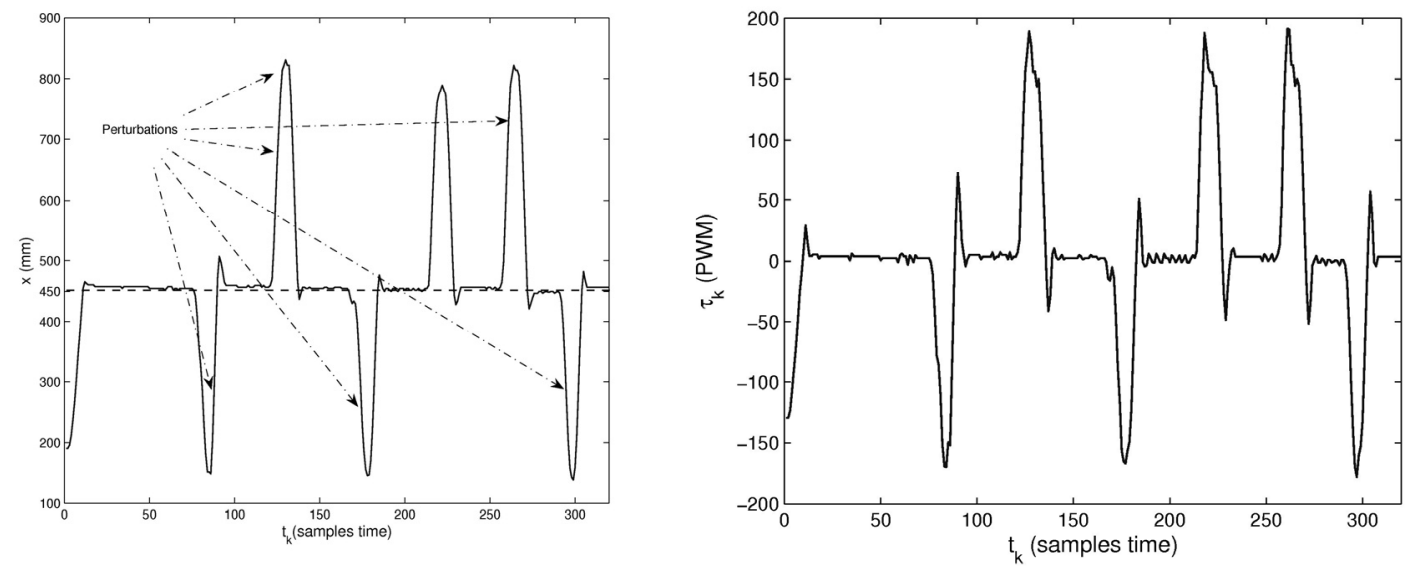

Figure 8. System response of the closed-loop system using a nonlinear control strategy based on saturation functions. 


\section{References}

[1] H.J. Sussmann, E.D. Sontag and Y. Yang, A general result on the stabilization of linear systems using bounded controls, IEEE Transactions on Automatic Control, 93(12) pp. 2411-2425, 1994.

[2] A. T. Fuller, In the large stability of relay and saturation control systems with linear controllers, International Journal of Control, 10 pp. 457-480, 1969.

[3] A.R. Teel, Global stabilization and restricted tracking for multiple integrators with bounded controls, Systems and Control Letters, 1992.

[4] E. N. Johnson and S. Kannan, Nested saturation with guaranteed real pole, American Control Conference, Brighton, pp. 497-502, 2003.

[5] Y. Yang, E.D. Sontag, H.J. Sussmann, Global stabilization of linear discrete time systems with bounded feedback, Systems \& Control Letters, 30, pp. 273-281, 1997.

[6] N. Marchand and A. Hably, Global stabilization of multiple integrators with bounded controls, Automatica, 41(12), pp. 2147-2152, 2005.

[7] Z. Lin and A. Saberi, Semi-global exponential stabilization of linear systems subject to input saturation via linear feedbacks, Systems \& Control Letters, 21, pp.225-239, 1993.

[8] Z. Lin and A. Saberi, Semi-global exponential stabilization of discrete-time systems subject to input saturation via linear feedbacks, Systems \& Control Letters,24, pp. 125-132, 1995.

[9] R. Lozano, J. Collado and A. Herrera, Semiglobal stabilization of continuous-time systems with bounded inputs, IEEE Transactions on Automatic Control, 44(12), pp 2318-2320, 1999.

[10] J. Collado, R. Lozano, A. Ailon, Semi-global stabilization of discrete-time systems with bounded inputs using a periodic controller, Systems \& Control Letters,36, pp. 267-275, 1999.

[11] P. Castillo, R. Lozano \& A. Dzul, Modelling and control of mini flying machines, Springer-Verlag, 2005. ISBN: 1-85233-957-8.

[12] Josep Forest and JoaquimSalvi.A review of laser scanning three-dimensional digitisers.Proceedings of the IEEE/RDJ Intl. Conference on Intelligent Robots and Systems.EPFL, Laussanne, Switzerland. October 2002.

[13] François Blais. Review of 20 years of range sensor development. Journal of Electronic Imaging. Vol. 13, No. 1, pp. 231-240. January 2004.
[14] Thomas P. Koninckx and Luc Van Gool. Real-Time Range Acquisition by Adaptive Structured Light .IEEE. Transactions on Pattern Analysis and Machine Intelligence. Vol. 28, No. 3, March 2006.

[15] David Acosta, Olmer García, Jorge Aponte, Laser Triangulation for Shape Acquisition in a 3D Scanner Plus Scan, Proceeding of the IEEE CERMA Electronics, Robotics and Automotive Mechanics Conference, Cuernavaca, México. pp. 14-19,2006.

[16] Marco A. García Romero, Antonio Cárdenas Galindo, Juan M. Rendón and Emilio J. González Galván. A Camera Calibration Method Applied to Vision Based Control of a Manipulator Robot. IEEE Latin American Robotic Symposium- IX Congreso Mexicano de Robotica (4th IEEE LARS 07 - IX COMRob 07).

[17] Danica Kragic and Henrik I Christensen.Survey on Visual Servoing for Manipulation.Research Report. Centre for Autonomous Systems, Numerical Analysis and Computer Science, Fiskartorpsv. 15 A. 10044 Stockholm, Sweden. Jan. 2002. http://www.nada.kth.se/danik/VSpapers/report.pdf

[18] Danica Kragic. Visual Servoing for Manipulation: Robustness and Integration Issues PhD. Thesis. CVAPNADA, Royal Institute of Technology, Stockholm, Sweden. 2001.

http://www.nada.kth.se/danik/thesis.pdf.gz

[19] Djamel Khadraoui, Guy Motyl, Philippe Martinet, Jean Gallice, François Chaumette. Visual Servoing in Robotics Scheme using a Camera/Laser-Stripe Sensor. INRIA Rennes. Internal publication No. 898.IRISA.ISSN 1166-8687. January 1995.

[20] A. C. Sanderson and L. E Weiss. Adaptive visual servo control of robots, In PughA. (editor). Robot Vision, I.F.S. Publications Ltd, pp. 107-116 1983.

[21] Anand Sanchez, Pedro Castillo, Juan Antonio Escareño Castro, Hugo Romero, Rogelio Lozano. Simple Real-Time Control Strategy to Stabilize the PVTOL Air- craft Using Bounded Inputs, European Control Conference, ECC'07, Kos, Greece, July 2007.

[22] Zhengyou Zhang, Flexible Camera Calibration By Viewing a Plane From Unknown Orientations, Microsoft Research, Redmond, WA 98052-6399, USA.

[23] Richard Hartley and Andrew Zisserman. Multiple View Geometry in Computer Vision. Second Edition. Cambridge Press 2003.

[24] Richard I. Hartley and Peter Sturm, Triangulation. Computer Vision and Image Understanding. Vol. 68, No. 2. November, pp. 146-157, 1997. 


\section{Authors' Biographies}

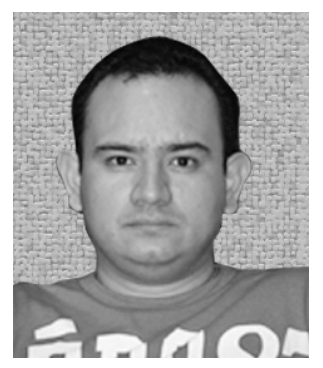

\section{Juan Manuel RENDÓN-MANCHA}

Juan Manuel Rendón-Mancha received a PhD degree in computer science from the Université René Descartes (Paris 5) in 2002. He earned a master's degree in artificial intelligence from the Université Pierre et Marie Curie (Paris 6 ) in 1998. He is an electronics engineer from the Instituto Tecnológico de La Laguna (1996). Since 2002, he has been associate professor at the Facultad de Ciencias of the Universidad Autónoma del Estado de Morelos, Morelos, Mexico. His research interests include computer vision for mobile and manipulator robots and analysis in biomedical images.

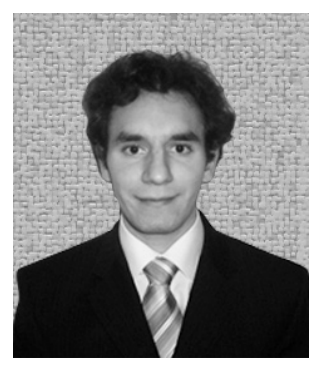

\section{Guillaume SANAHUJA}

Guillaume Sanahuja obtained the B.S. degree in industrial systems engineering in 2006 from the Ecole Nationale Supérieure d'Arts et Metiers, Paris, France. He received the M. Sc. Degree in robotics from the Université Paris VI, France in 2006 and the Ph. D. degree in automatic control from the University of Technology of Compiègne, France, in 2010. At the moment, he is a researcher engineer at the French National Research Foundation (CNRS), in the Laboratory Heudiasyc, at the University of Technology of Compiègne, France. His research topics cover real-time control applications, embedded systems, embedded vision and UAVs.

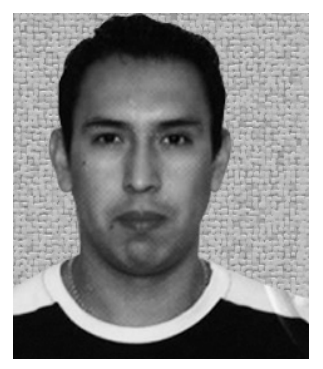

\section{Pedro CASTILLO}

Pedro Castillo obtained the B.S. degree in electromechanical engineering in 1997 from the Instituto Tecnológico de Zacatepec, Morelos, Mexico. He received an M.Sc. degree in electrical engineering from the Centro de Investigación y de Estudios Avanzados (CINVESTAV), Mexico, in 2000, and a Ph.D. in automatic control from the University of Technology of Compiègne, France, in 2004. At the moment, he is researcher at the French National Research Foundation (CNRS), in the Laboratory Heudiasyc, at the University of Technology of Compiègne, France. His research interests include real-time control applications, nonlinear dynamics and control, aerospace vehicles, vision, and underactuated mechanical systems 


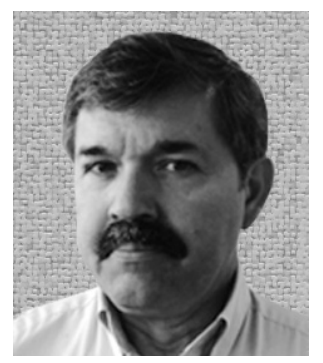

\section{Rogelio LOZANO}

Rogelio Lozano received the B.S. degree in electronic engineering from the Instituto Politécnico Nacional de México in 1975, the M.S. degree in electrical engineering from the Centro de Investigación y de Estudios Avanzados (CINVESTAV), Mexico, in 1977, and the Ph.D. in automatic control from Laboratoire d'Automatique de Grenoble, France, in 1981. He joined the Department of Electrical Engineering at the CINVESTAV, Mexico, in 1981, where he worked until 1989. He was head of the Section of Automatic Control from June 1985 to August 1987. He has held visiting positions at the University of Newcastle, 1987. Since 1990, he has been CNRS research director at the University of Technology of Compiègne, France, and since 1995, head of the CNRS Laboratory Heudiasyc (Heuristique et Diagnostic des Systèmes Complexes). His research interests are in adaptive control, passivity, nonlinear systems, underactuated mechanical systems, and autonomous helicopters. 